\title{
HIGH-PRECISION APPLICATION OF GPS IN THE FIELD OF REAL- TIME EQUIPMENT POSITIONING
}

\author{
François PEYRET, David BÉT AILLE, Gaëtan HINTZY \\ LABORATOIRE CENTRAL DES PONTS ET CHAUSSÉES - NANTES - FRANCE \\ B.P. 19 - 44340 - BOUGUENAIS - FRANCE \\ Tel : $+33.240 .84 .56 .23 /+33.240 .84 .59 .40$ \\ E-mail : Francois.Peyret@Icpc.fr / David.Betaille@ Icpc.fr
}

\begin{abstract}
In the frame of its research about real-time positioning and control of road construction equipment, the "Laboratoure Central des Ponts et Chaussées, has carried out in 1996 a study to know more about the actual vertical accuracy that a $R T K^{I} G P S^{2}$ could reach, under worksite conditions.

This study has widely used the dedicated testing facility SESSYL, built to perform high-accuracy and realtime evaluation tests on positioning systems. It has been performed in collaboration with the French road contractor COLAS and the "Ecole Supérieure des Géomètres et Topographes »(ESGT).

First, is proposed an adapted geodetic transformation procedure, compatible with the high accuracy of the requirements. Then are presented the main results of a special SESSYL tests program, where the impacts of several influencing parameters on the vertical accuracy have been carefully examined.

The core part of the paper is the analysis of the typical RTK GPS set of data, from which we have tried to extract two different components : a high-frequency noise, rather easy to filter, and a low-frequency bias. This bias, given its good repeatability, can be modeled and used in prediction to improve in real-time the raw accuracy of the data.

As a full scale validation of our study, is finally described a site experiment, carried out this time on a real piece of equipment (an asphalt paver) during a real work site.
\end{abstract}

\section{Introduction}

The recent arrival on the market of real-time positioning systems is opening the door to a totally new methodology for carrying out the construction work sites. Thanks to these new systems, we can now overcome the weakness existing on construction sites today - e.g. the huge gap between the design phases which are highly

\footnotetext{
${ }^{1}$ RTK : Real-Time Kinematic

${ }^{2}$ GPS : Global Positioning System
}

computerized and the work site itself where all numerical data is reduced to wooden grade stakes [1].

The LCPC is a public applied research center dealing with all the civil engineering activities, from soil mechanics to road construction and maintenance, including the environmental and safety aspects of the transportation infrastructures.

Given its historical background, its activity about new construction methods, and in particular real-time positioning and control of the equipment, is focused upon road construction machines.

Many technological answers exist to the positioning problem of these machines, depending mostly upon the type of work.

Roughly, we can divide the public works construction machines into three main groups, which differ according to requirements in terms of positioning :

- earth-moving and mining equipment, e.g. the big earthworks machines like shovels, drills, scrapers, dozers. excavators, etc.

- so-called «surfacing equipment », that is to say the machines which moves on the surface of the ground, without noticeable changes in height : compactors, cement spreaders, mixers, etc.

- so-called "profiling equipment»», that is to say the machines which modify the profile of the work site by addition or removal of material : pavers, autogrades, milling machines, etc.

The paper will deal with the positioning of the third group of equipment which is the most demanding one in terms of accuracy, especially in terms of elevation $(\mathrm{Z} \mathrm{co}-$ ordinate).Currently, to solve this problem, optical solutions (laser planes, automatic theodolites...) are chosen, given their high accuracy.

GPS technology could lead to very competitive solutions, as far as the required accuracy is reached and the shadowing problems are solved. Their main and quite significant advantage in terms of ease of installation on the site being actually very attractive for the end users, e.g. the civil-engineering construction companies. 
The new real-time kinematic GPS, capable of providing the 3 co-ordinates of any mobile point with a centimeter accuracy, at a frequency of one $\mathrm{Hz}$ or more, has seemed to us particularly interesting to be studied for this new application

\section{Requirements of the paver in terms of absolute positioning}

\subsection{In terms of elevation} finisher.

Figure 1 shows schematically the asphalt paver or

This machine moves very slowly (some meters per minute), always forward, but has to spread a very smooth and precisely leveled layer of material. The material is brought from the hopper to the rear of the tractor by a conveyor and spread on the ground by a distribution screw in front of the floating screed. The screed is also equipped with compaction and vibration mechanisms to better pre-compact the material.

This level is directly set by the position of the tool (a so-called «floating screed » attached to the tractor at two articulations called «tow-points » by the intermediate of two «leveling arms»). The equilibrium of the screed, free of rotating around the tow points depends upon several parameters. Two of them are the lengths of the two hydraulic actuators (moving up and down the tow points) but the others are internal parameters from both equipment (vibrators, tampers, speed...), and material (composition, viscosity...).

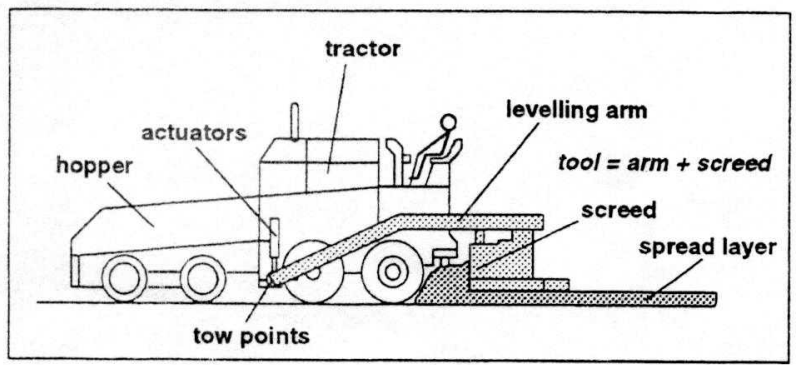

Fig. 1 : Schematic representation of the asphalt paver

This high accuracy in terms of level comes from the contractual tolerances in level and cross-slopes currently required for the upper layers of the pavement, typically \pm $3 \mathrm{~cm}$ for the sub-base, $\pm 2 \mathrm{~cm}$ for the base, $\pm 1.5 \mathrm{~cm}$ for the binder course and $\pm 1 \mathrm{~cm}$ for the wearing course.

The absolute control in elevation is essential for the spreading of the sub-base (sometimes base) layer, in order to respect the altitudes and cross-slopes prescribed by the design.

This control is currently performed by using string lines (also called « wire ropes ») installed along the road and mechanical deviation sensors. These wires are expensive to install, dangerous for the workers and generate problems for worksite truck traffic.

Sometimes, on big worksites, the string line is replaced by a plane generated by a rotating laser beam and the sensors are photo-cells [2]. This device provides only a reference plane and is not really convenient for controlling the tool along an undulating profile, as most of the roads are...

GPS, compared to these two techniques, could bring significant advantages:

- the easiness of use and of installation (only one base station would be necessary for a few kilometers long worksite),

- the highly automated process for the computing of the position which guarantees a very good reliability (almost no more manual settings and checking procedures, sources of numerous human errors),

- the providing of the three co-ordinates all together, with a perfect coherence between them.

Some other control methods are used when the previous layer is supposed to be correctly leveled and when the essential parameter to control for the current layer is the thickness. In this case, the absolute positioning using GPS is not so much interesting compared to other methods using on-board measurements between the tool and the ground.

\subsection{In terms of plane position}

It is also essential to get the plane co-ordinates $\mathrm{X}$ and $\mathrm{Y}$, for two main reasons :

- it can be interesting to envisage a plane navigation system for the tractor, which can be totally automatic or in the form of a smart man-machine interface for the driver,

- the pane co-ordinates are necessary for computing the current abscissa of the machine, in order to provide the elevation control system with the reference altitude and slope corresponding to this abscissa.

Nevertheless, the accuracy needed for these two functions (around $\pm 10 \mathrm{~cm}$ ) is not very stringent compared to the RTK GPS accuracy, so we have focused our study on the elevation accuracy.

\section{Localization using Real Time Kinematic GPS}

\subsection{GPS}

GPS is used in many applications : sailing, aerial and terrestrial navigation, geodesy, etc.[3]. The specifications in terms of accuracy for these different applications are not the same of course : from less than a centimeter to around a hundred meters. To get centimetric accuracy, a single GPS receiver is not sufficient, we must operate with a pair of receivers : it is 
called «differential GPS» (and not «absolute» or «natural »).

Differential GPS consists in measuring distances with several common satellites from a pair of receivers. The distances are computed from GPS waves codes C/A or $\mathrm{P}$ : so computed distances are called pseudo-distances and the typical accuracy in this mode is about 1 meter (« differential mode $»)$.

The distances can also be computed from carrier phases of the waves, on L1 frequency or on L1 and L2 frequency (the initialisation time is divided by ten if both L1 and L2 are used). The positions are known then with a centimetric accuracy («kinematic mode»). The phase measurements permit reduction in the influence of ephemeris, waves propagation, clocks drifts between satellites and receivers...

The result of differential GPS is a vector from a static receiver (base), to other static or mobile receivers (rovers). The vectors can be computed either in postprocessing, or in real time if a radio link (modem) exists between base and rovers ( real-time kinematic mode »). A vector is computed at least every second $(1 \mathrm{~Hz})$ in this mode, the most recent instruments perform high frequency rates, up to 5 or $10 \mathrm{~Hz}$.

\subsection{Geodetic references}

To keep the vector centimetric accuracy, its transformation from GPS reference system (WGS 84) to local reference system on the works site must be very precise.

\section{- Local systems}

$\mathrm{CAD}$ road projects are given in plane projection and in vertical projection (along gravity). In these kind of local references, one particular point, depending on the local reference, has $\mathrm{X}$ and $\mathrm{Y}$ co-ordinates equal to zero. A zero level is also defined. Planimetry and altimetry are not necessarily included in the same reference : it is not a $3 \mathrm{D}$ reference, but what we could call a $2 D+1$ reference.

In France, the local reference is composed of Lambert projection (conic and conformal projection) and the NGF 69 «Nivellement Général de la France» altitude (vertical projection along gravity). Lambert $X$ and $\mathrm{Y}$ are equal to zero in Paris, and the sea level in Marseilles define the zero level. In other countries, Mercator (cylindrical and conformal projection) is used.

The equipotential surface of earth's gravity field, corresponding approximately to the mean sea level is called the «geoid». It is a non-mathematical surface, quite hard to define accurately everywhere.

- Global systems

On the contrary, WGS 84 is a $3 \mathrm{D}$ global reference, defined from an ellipsoid. Many ellipsoids exist, attached to different reference systems. They are of course perfect mathematical surfaces, defined by two constants, which do not fit the geoid. Any point can be located using three cartesian (X, Y, Z) or three geodetic (I, j, h $\left.{ }^{3}\right)$ coordinates. $(\mathrm{X}, \mathrm{Y}, \mathrm{Z})$ to $(\mathrm{l}, \mathrm{j}, \mathrm{h})$ transformation, or inverse, are determined by mathematics formula, taking into account the geometrical parameters of the ellipsoid

- GPS

The GPS data are basically computed in the global reference WGS 84 and must be transformed from WGS 84 to local reference. The general transformation formulas cannot be applied abruptly for that, because of two main sources of errors :

- non independence and non constant accuracy of the terrestrial survey (contrary to spatial survey) which has generally been done to set the monuments used on the works sites,

- non constant distance between WGS 84 ellipsoid and geoid (gravity).

We propose here a method to transform the GPS data, with a determination of new local parameters instead of general parameters.

The general parameters are sufficient for applications needing about $5 \mathrm{~cm}$ accuracy. But we cannot accept it. Our method consists in surveying several monuments near the works site and compute their coordinates in the spatial reference (WGS 84). The method requires differential GPS and post-processing.

The surveyed monuments must of course also be known initially in the local reference.

Enough monuments near the site, that is to say at least 3 monuments (for least mean square) in every area of $5 \mathrm{~km}$ round, will allow us to compute a series of local parameters all along the works site. A gradient of these parameters is used between consecutive areas.

Besides, the comparison of local geoid altitude and WGS 84 ellipsoid altitude gives us the way to correct and adapt the geoid-ellipsoid difference $N$, not considered as a constant anymore. A gradient of $\mathrm{N}$ can be applied in the areas and from one area to the other.

The method presented here, whose necessity appears evident for centimetric GPS measurements and applications, is currently applied on the SESSYL site (Localization Systems Test Bed) and used to compare GPS and reference trajectories performed with this equipment.

In France, the National Geographic Institute is by now setting out new monuments using GPS (called RGF) which should be very useful in our method (we have used some of them for our experimental worksite). The monuments are every $20 \mathrm{~km}$ and are given in WGS 84 with an accuracy of one centimeter. This accuracy is available everywhere in France.

\footnotetext{
3 « I stands for longitude, « $\mathrm{j}$ » for latitude and « $\mathrm{h} »$ is the height of the point above the ellipsoid
} 


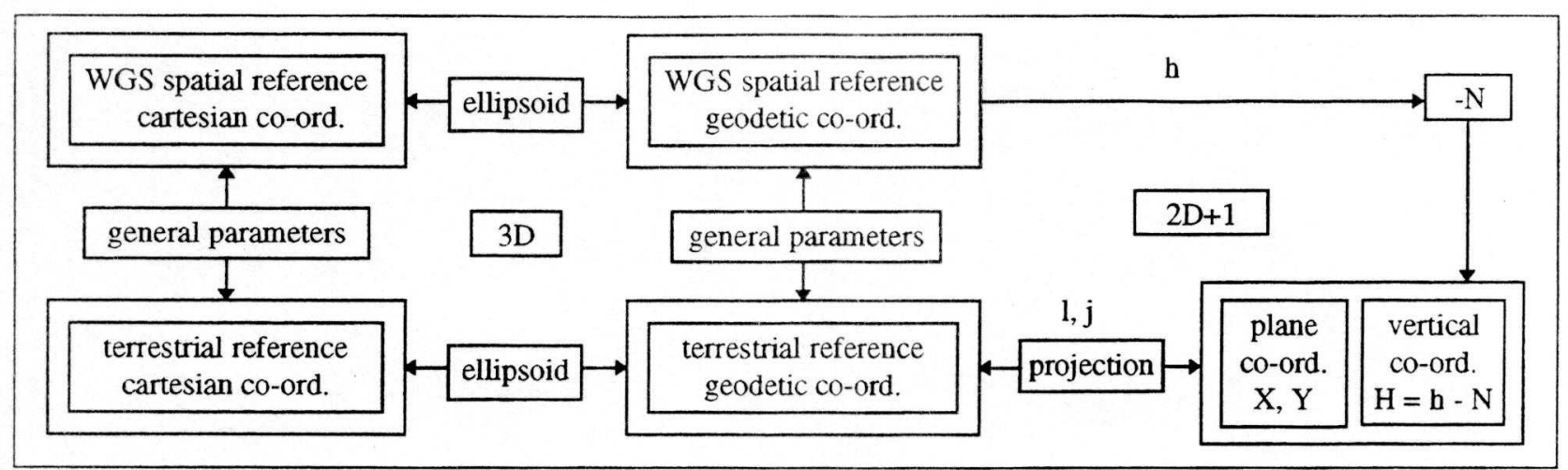

Figure 2 : geodetic transformations between GPS and a local work site reference system

\section{Influence of several parameters on the vertical co-ordinate accuracy}

Once solved the problem of the data transformation from GPS to the works site reference, we have studied the influence of several parameters on the vertical accuracy.

SESSYL is a new facility of the LCPC Nantes center built to support the researches and assessments carried out there in the field of site robotics and dynamic positioning of construction equipment [4]. It has mainly contributed to our tests. Four categories of tests were carried out and we present here a statistic analysis of the differences between the RTK GPS trajectory and the SESSYL reference trajectory obtained by internal sensors.

The GPS receivers which have been used for this tests are TRIMBLE $7400 \mathrm{MSi}$

\section{'A' category tests}

Their objective was to improve our knowledge of the vertical accuracy, in terms of stability, reliability, accuracy and signature of the signal. Tests have been carried out all day long for several days. The movement of SESSYL was uniform at $0.1 \mathrm{~m} / \mathrm{s}$ speed (like a finisher).

The complete set of ' $A$ ' tests represents 14 hours of measurement, giving about 50000 GPS points (at $1 \mathrm{~Hz}$ ). It is a good indicator of the accuracy of GPS with regard to the variations of the constellation all through the day. Each revolution of the carriage of SESSYL on its track is subdivided into 4 sections and the data are processed to provide one point per section on the «mean versus standard deviation » diagram.

We can note an exactitude better than $\pm 25 \mathrm{~mm}$. with a standard deviation under $15 \mathrm{~mm}$.

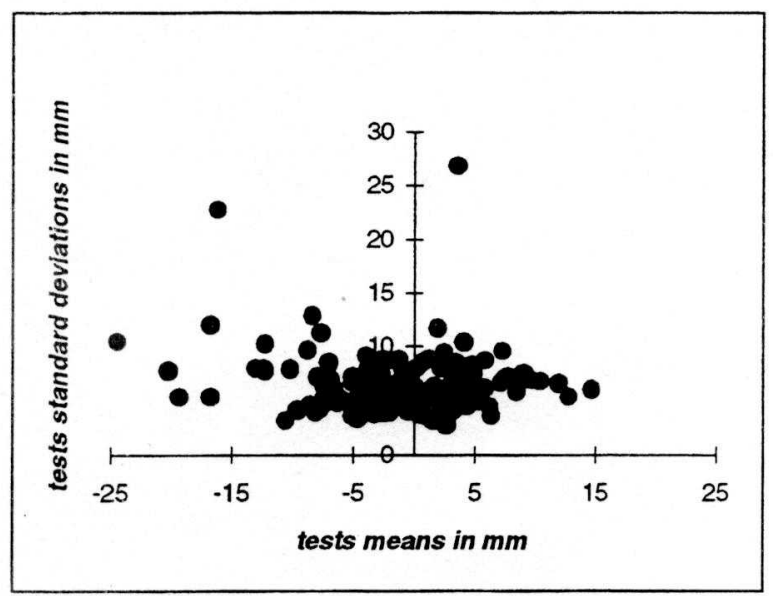

Figure 3 : SESSYL tests without platform movements, section by section

\section{' $B$ ' category tests}

To 'A' tests conditions were added different kind of vertical movements of the antenna on the SESSYL platform :

- slow vertical movement : slopes of $2 \%$ during 10 $\mathrm{m}$, and of $8 \%$ during $2.5 \mathrm{~m}$ all along the tests,

- rapid vertical movement : square steps of $\pm 30 \mathrm{~cm}$ every $5 \mathrm{~m}$ during the tests.

On the figure 4 are presented the points corresponding to these tests, together with the points corresponding to the ' $A$ ' tests, computed this time revolution by revolution

No significant differences can be noted between the two families of points, showing that the vertical movements have no influence on the accuracy. 


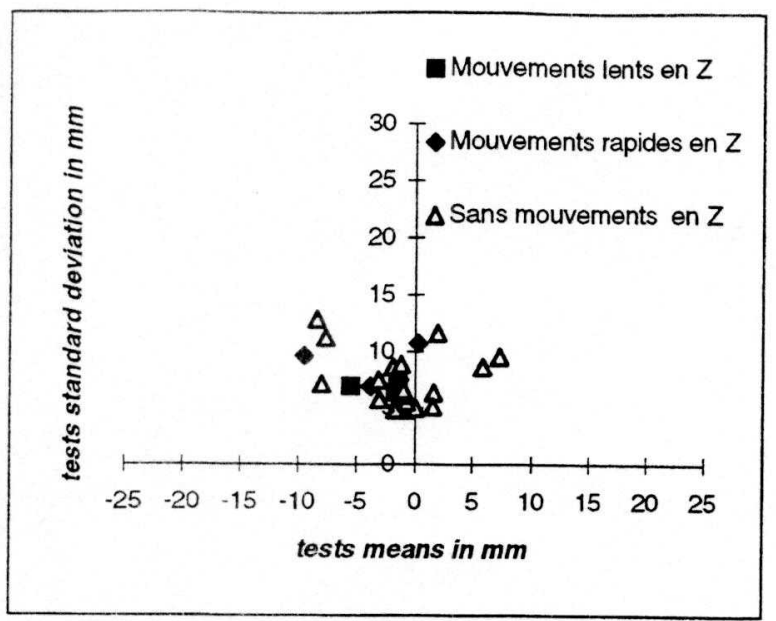

Figure 4 : tests with and without platform movements

\section{' $\mathrm{C}$ ' category tests}

Here, the parameter analyzed is the length of the base-line. Two families of points are represented : those corresponding to a $120 \mathrm{~m}$ long base-line and those corresponding to a $4 \mathrm{~km}$ long base-line.

We can conclude that, from $100 \mathrm{~m}$ up to $4 \mathrm{~km}$, there is no significant change in the accuracy.

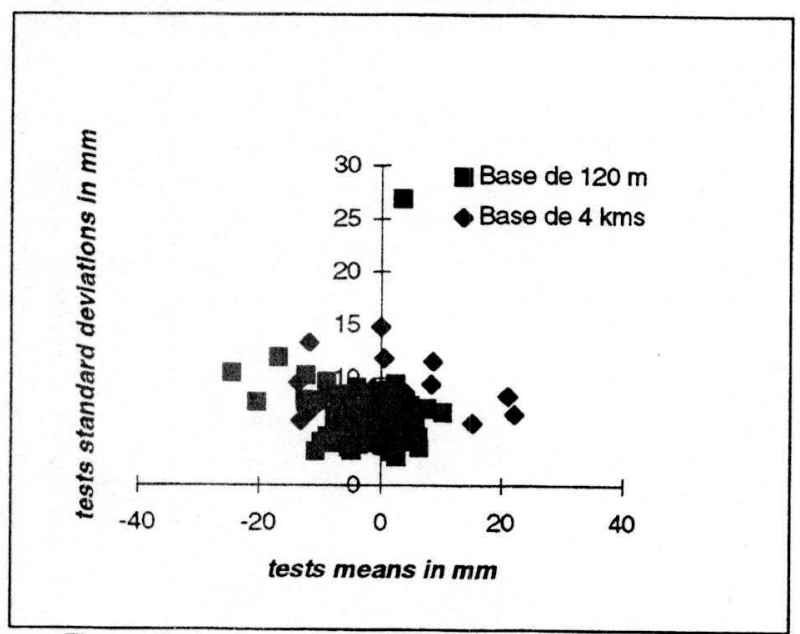

Figure $5:$ tests with two different base-line lengths

\section{'D' category tests}

They have been performed to analyze the accuracy when the GPS is recovering the RTK mode after a complete mask. (We can simulate this mask with SESSYL carriage running under a $6 \mathrm{~m}$ long tunnel.) The tests have been performed with the two different baselines.

We have noted here that the full accuracy is immediately reached as soon as the RTK mode is recovered.

These tests have also been used to analyze the « onthe-fly » initialization time. This time appears very variable, with no correlation (strangely...) with the number of available satellites.

According to the above table, it seems that, on average, this time increases with the base-line length, but the shortest time $(10 \mathrm{~s})$ was observed on a $4 \mathrm{~km}$ long base-line !...

\begin{tabular}{|cc|}
\hline base-line & mean initialisation time after a mask \\
$120 \mathrm{~m}$ & $64 \mathrm{~s}$ \\
$4 \mathrm{~km}$ & $90 \mathrm{~s}$ \\
\hline
\end{tabular}

\section{Signal analysis of GPS data}

\subsection{In the time/space domain}

In our study, GPS is intended to be used to measure the level of the floating screed in order to control it : so, we have to analyze the GPS data according to the road defects (disturbances) which must be corrected.

These defects are defined in terms of waves lengths and amplitudes. We here refer to the "Analyseur de Profil en Long " or "APL " (Longitudinal Profile Analyzer) developed at the LCPC. In France, waves lengths are currently classified in :

- short waves lengths $(0.7$ to $2.8 \mathrm{~m})$,

- medium waves lengths (2.8 to $11.2 \mathrm{~m}$ ),

- long waves lengths (11.2 to $44.8 \mathrm{~m}$ ).

Inside its frequency domain of validity, the APL allows to quantify the eveness with a note, for every waves lengths class. We can consider that defects with amplitudes up to $5 \mathrm{~cm}$ can be met, especially on base or sub-base layers.

Figure 6 shows 2 typical sets of data, recorded at 2 different times, during 12 hour periods.

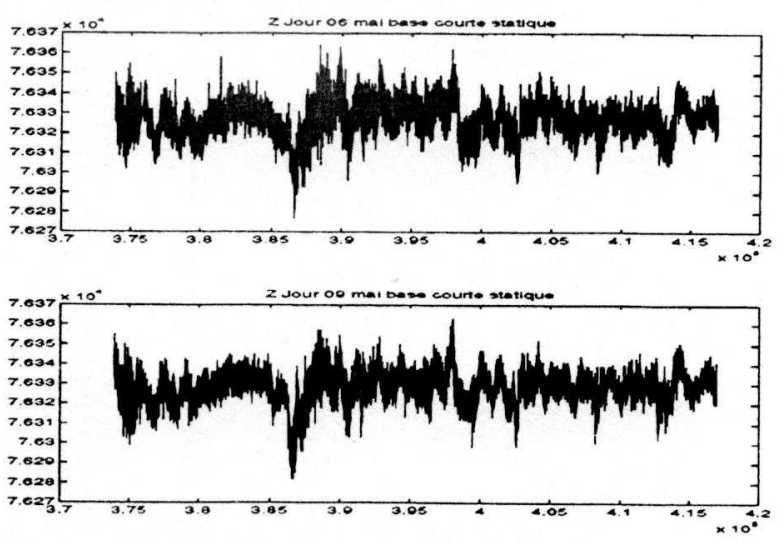

Figure 6 : typical RTK GPS data

The GPS data show oscillations whose waves lengths and amplitudes ( $\pm 2.5 \mathrm{~cm}$ for the receivers used) are exactly of the same scale as road defects. Indeed, the duration of GPS oscillations are $30 \mathrm{~s}$ to $10 \mathrm{~min}$, with consequent waves length of 1.5 to $50 \mathrm{~m}$ considering a 
paver speed between 3 to $5 \mathrm{~m} / \mathrm{s}$. The oscillations cover all the frequency domain of the APL.

Let us now introduce what we call GPS «noise » and « bias ».

We consider that the GPS data are separated into 2 domains of frequency by the central value $0.05 \mathrm{~Hz}$ : noise is above $0.05 \mathrm{~Hz}$, bias is below $0.05 \mathrm{~Hz}$. This cutting frequency is equivalent to a $20 \mathrm{~s}$ period, or to $1 \mathrm{~m}$ wave length in the space domain, at a finisher's speed. It is close to the shortest wave length which can be measured with the APL.

A visual analysis of the GPS data in the time domain shows that the amplitude of noise is $5 \mathrm{~mm}$ maximum. This is approximately the value of the standard deviations computed on the tests and presented above. The noise contains no information relevant to position and is definitely considered as white noise.

On the contrary, bias has centimetric amplitudes, and represents the oscillations, or drift of GPS data around the mean value of localization. This drift is called

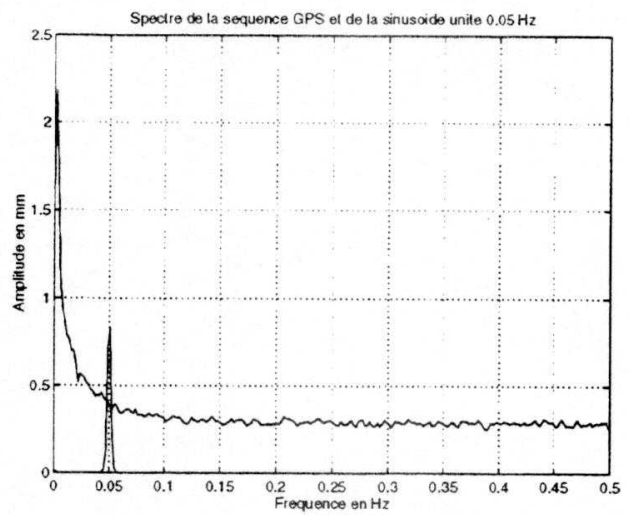

«bias». (Note that, in our definition, the bias is not constant but a function of time).

A consequence is immediate: the control system using GPS data must :

- filter the noise,

- model the bias, that is to say predict it

We foresee the major issue : how can GPS bias and road defects can be separated from each other?

\subsection{In the frequency domain}

Figure 7 shows the results of a spectral analysis. The GPS data collected in this session are GPS kinematic data, collected on a static point, during a $12 \mathrm{~b}$ session.

The left-hand figure superpose the Fourier spectral analysis of the GPS data and the same analysis for a 0.05 $\mathrm{Hz}$ unit sinusoid. The right-hand figure shows the GPS data when noise has been filtered, using an ideal filtering process : Fourier transform, cutting of all the frequencies above $0.05 \mathrm{~Hz}$ and then inverse Fourier transform ( «Fourier filter »).

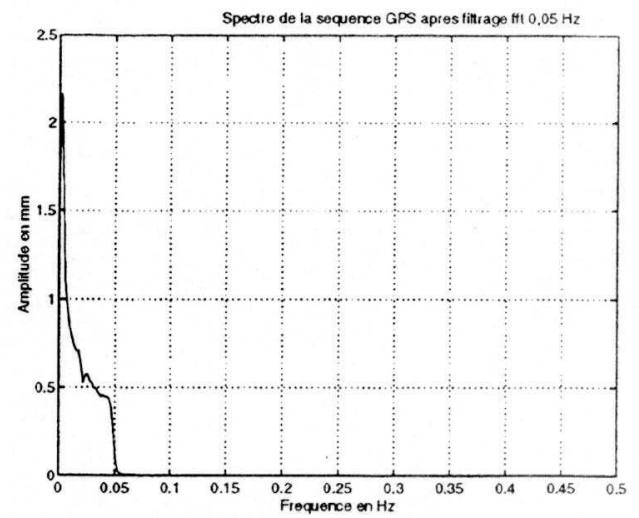

Figure 7 : spectral analysis of typical GPS data

\section{The filtering of the noise}

A filter to get id of the noise is necessary. We tried to find a real-time filter approaching as well as possible the ideal Fourier transform and inverse filter (which is not real time of course !). Particularly, we had to minimize the phase shift introduced as a consequence of the real time.

Several filters were envisaged :

- auto-regressive filters (like Normal Least Mean Square), but unused because the data cannot be centered,

- sliding mean (mean of the current point with $n$ precedent points), but unused too because of its phase difference,

- sliding regression (extrapolation on the current point of the regression line of the $n$ precedent points),

- median filter (median point in a pack constituted of the current point and the n previous).
We went deeper in the study of sliding regression and median filter. These two filters were compared to the ideal Fourier filter, considered as a reference. We tried to minimize the standard deviation of the difference between the filtered GPS data on the one hand, and the reference on the other hand. This comparison was computed on the $12 \mathrm{~h}$ static session. The parameter to choose was the horizon of the filter.

We finally chose the horizon $\mathrm{n}=3$ median filter : it is quite satisfactory and it has a phase difference less important than upper horizon median filters and provides the same efficiency.

Moreover, this filter is easy to initialize which is very interesting for GPS subject to masks. It also suppresses aberrant data without smoothing the information.

The efficiency of this filter can be compared to the ideal filter one on figure 9 . 


\section{The processing of the bias}

The bias is a much more difficult problem to solve...

We do need a bias model in order to know before their occurrence the tendencies of the GPS data and to be able to separate the surface defects from the drift of GPS itself.

First we tried to find a correlation between the bias and the satellites geometrical configuration. Our analysis has shown no significant correlation between the bias and the PDOP (Position Dilution of Precision) or the VDOP (Vertical Dilution of Precision).

Then we paid attention to series of tests where the sky plot was nearly the same in order to detect a repeatability...

\subsection{A model of GPS bias}

We started our study by the analysis of two sets of GPS static data, filtered by the "Fourier " filter, and collected at two different days (figure 6). The two measurements were performed with a short base-line of a hundred meters, under exactly the same sky plots (same satellites and same angles), at exactly the same GPS time, but with a $3 \times 86164 \mathrm{~s}$ time period between them. The time of $86164 \mathrm{~s}$, equal to 23.93 hours (one « GPS day ») corresponds exactly to two revolutions of the GPS satellites.

The comparison of recorded data shows a clear repeatability of the GPS bias. The difference is unbiased, with a standard deviation is about $1 \mathrm{~cm}$ and a signature very close to a white noise one.

Other series of tests showed that the GPS measurements were indeed repeatable, but the quality of this repeatability depends on given parameters.

The parameters we have studied the influence of are:

- the PDOP,

- orientation of the antenna,

- the period of repeatability, that is to say the number of «GPS days » between the two sets of data,
- the distance between the two receivers, that is to say the length of the GPS vector.

PDOP

The PDOP is important. Here, it seems to be a very good indicator of the repeatability of the GPS data. When the constellation is not exactly the same on a pair of recorded sets, the difference is still biased.

Orientation of the antenna

The orientation of the antenna, changing when SESSYL carriage performs a revolution, has apparently no influence.

\section{Difference in time}

As the number ( $\mathrm{n}$ ) of « GPS days » between the two sets increases, the worse is the repeatability. The limit not to exceed seems to be four days.

\section{Difference in space}

On long baselines, the signature of the data differs lightly from the reference record because the sky plot geometries are not so similar, especially when satellites transitions occur. The data differs more in amplitude than in phase, which maybe could be explained by multi-path effects.

Note: the antennas used during these tests had no deflector : they were sensitive to multi-paths.

\subsection{How to use this model}

Actually, our real issue is to correct a dynamic set (a static base and a mobile rover) with a static one, obtained $n$ »GPS days » before.

Figure 8 presents the synopsis of the method we have tried.

A perfectly filtered set of static data, obtained $n$ days before, is synchronized with the real-time raw measurements using GPS time. This bias (filtered through a «Fourier » filter) is subtracted from the raw measurements (filtered through a real-time median filter), to obtain the corrected data to be used by the on-board control system. The process is full real-time.

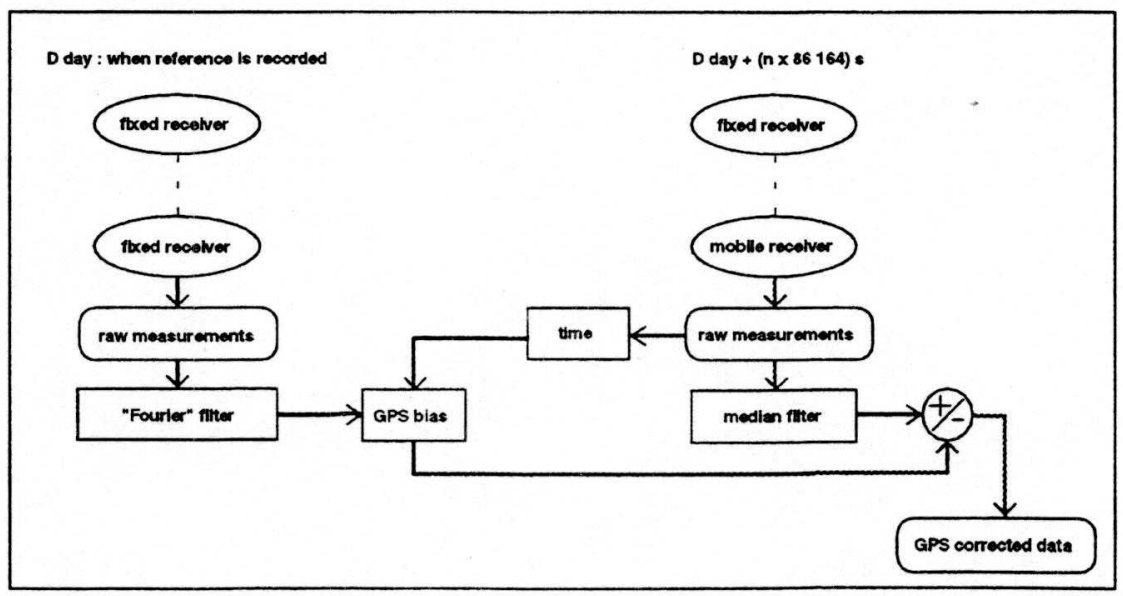

Figure 8 : synopsis of the proposed method to improve the real-time accuracy 
The efficiency of such a method is illustrated by figure 9. The upper curve is the raw real-time dynamic data set, the middle one is the bias, the last one is the corrected set of data, obtained by difference.

The mean value is improved from $-4.8 \mathrm{~mm}$ to -1.5 $\underline{\mathrm{mm}}$ and the standard deviation is improved from 13.7 $\mathrm{mm}$ to $7.5 \mathrm{~mm}$. Roughly, we can say this method improves the accuracy by a factor of two, keeping the measurements in an interval of $\pm 1 \mathrm{~cm}$ (at $1 \sigma$ ) around the true data
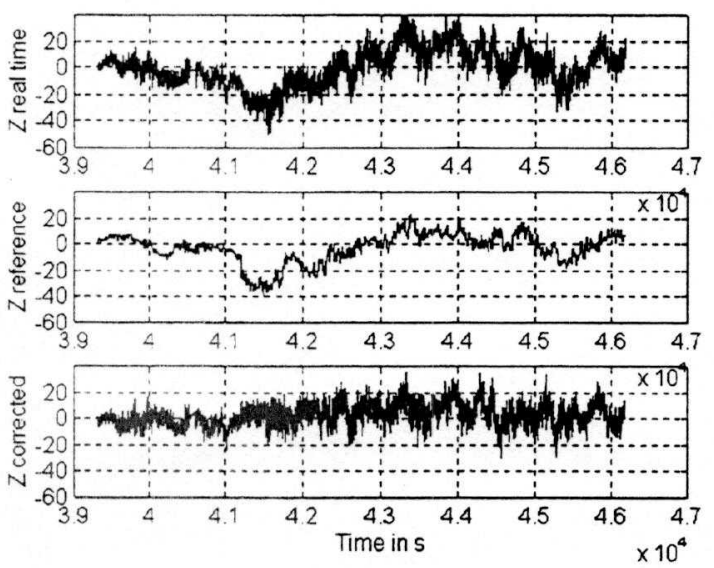

Figure 9 : efficiency of the bias correction on a dynamic set of data

\section{Full-scale experiment}

We have tested the method described above on a real work site.

These tests should complete our investigations on the limits of the method and warn us of some practical problems which could not have been foreseen on SESSYL site (potential masks or multi-path effects in particular).

This experiment has been carried out on a paving worksite (asphalt binder course), $100 \mathrm{~km}$ south from Paris. We have used three receivers of the same type, in the same time : one of them was the static base, the other was a static rover which role was to record the bias, the last one was the mobile rover on the finisher. The elevation of the screed was controlled by a traditional system, using a mechanical sensor and a leveling beam (thickness control). A surveyor theodolite was used as a reference instrument, for two measurements : the " real » profile of the spread layer just behind the paver (before compaction) and the « real » elevation of the GPS antenna, theoretically equal to each other.

Figure 10 presents a schematic view of all the instruments which have been used on the worksite.

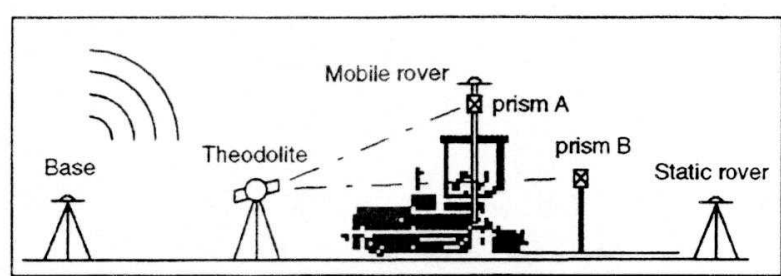

Figure 10 : the instruments used on the site

Firstly, we applied the geodetic transformation method already proved to be efficient on SESSYL site in order to guarantee no geodetic bias in our data.

Then, we applied the bias correction method, with the following difference : this time, the bias model was not established using previous measurements, but using the perfectly synchronized measurements between base and static rover. So, we got rid of the time difference influence. All that remained was the space difference influence.

The final corrected data were however computed by post-processing.

Both raw and corrected GPS data have been compared to the theodolite data.

The analysis of the results has shown that our method was efficient on some sections of the road but totally inefficient on others. The limits of the method appeared clearly : the bias model is useless if constellation, horizon, multi-paths or masking conditions are different from one antenna to the other.

But when good conditions are satisfied, the bias is corrected and the required centimetric accuracy finally reached.

\section{Perspective}

We foresee a very interesting perspective to our work, connected to the accuracy of RTK GPS measurements. We called it : « double-base » survey.

Double-base survey consists of using simultaneously 3 GPS receivers ( 2 vectors), in real time.

We indeed believe that it is possible to evaluate the GPS bias on a pair of static receivers, and to use it immediately on a mobile receiver (or several ones). The static receivers must be located on known positions. One of them can work in RTK with the mobile(s) dynamic vector(s) while surveying the static vector.

A supplementary radio link would be necessary to apply this double-base survey (transmission of the bias evaluation)

Figure 11 presents what could be a full real-time processing of the data using the « double base survey ».

But, actually, it is an external solution from the GPS signal processing which is proposed here : we have never use satellites measurements but GPS data already computed. From a basic point of view, a more efficient solution is expected from the GPS manufacturers 
themselves. We are very hopeful regarding new GPS RTK generation, that could meet our requirements in

terms of accuracy, using a double-base survey in real time.

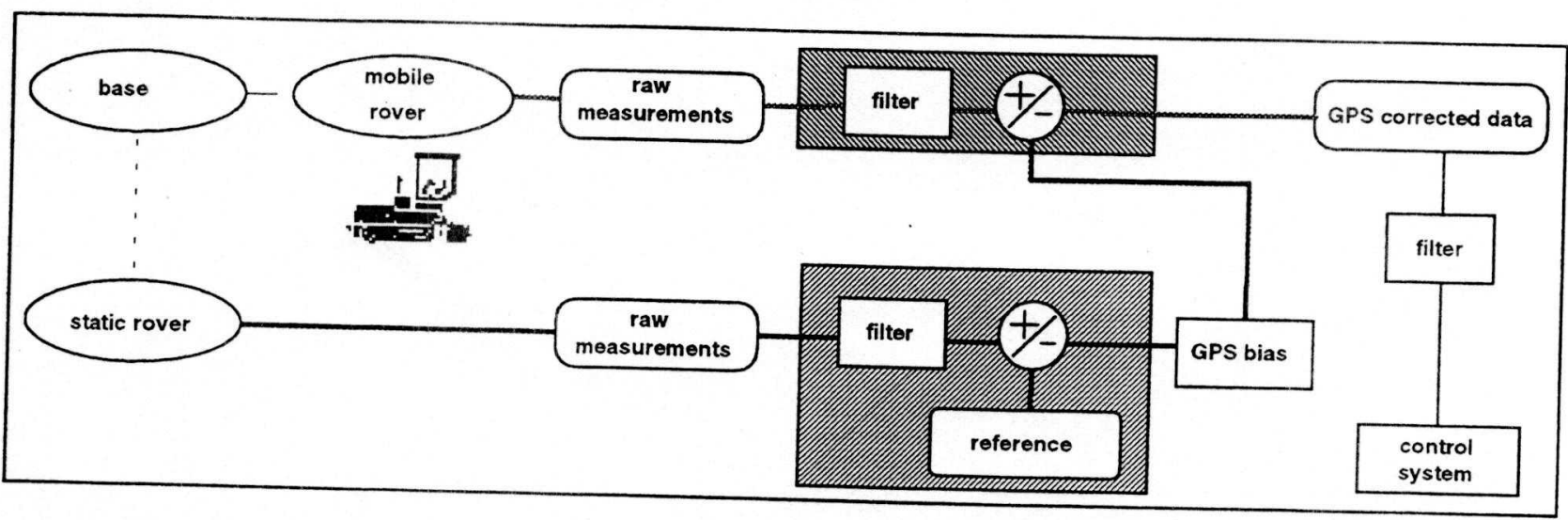

Figure 11 : double-base survey applied to a machine control in real-time

\section{Conclusion}

This study has been decided to bring an answer to this "burning" question : "can GPS be used for the elevation control of the profiling equipment's tool such as the screed of a paver? » It has been carried out with one of the best RTK GPS material existing at this moment and has lead to the main following results.

- In terms of accuracy, despite the impressive results, it seems now obvious that the raw measurements are not accurate enough so far to be used by the control systems. This fact is due to the bias error, or "drift», we have pointed out and which is obviously correlated to the constellation plot, through some local phenomena such as multi-path effects.

- This accuracy, first achieved on the particular and well-controlled SESSYL site, should not be too much degraded by the real worksites conditions, as long as some basic precautions are taken.

- An important fact we have pointed out is that this drift is repeatable, within given limits in terms of time ad space, and appears quite independent of the movement of the mobile. A static RTK set of measurement shows approximately the same error signature as a dynamic one which is achieved in a close neighborbood.

- This repeatability can be used to improve the raw accuracy of the measurements, improving it, in given cases, by a factor of two. A simple solution, external from the GPS signal processing, is proposed but a more efficient solution is expected from the GPS manufacturers.

As a final conclusion, the possibilities of improving the accuracy by an appropriate real-time processing added to the improvements which will surely be brought soon by the manufacturers themselves, allow us to think that GPS technology would achieve in the near future the accuracy needed for elevation positioning of road construction equipment.

\section{References}

[1] Peyret F., Philippe H.

Towards Computer Integrated Road Construction $9^{\text {th }}$ ISARC, Tokyo, pp. 859-868, June 1992

[2] Legentil H., Martineau Y. A83: an innovation in the automatic guiding of paver grading Revue générale des routes et aérodromes $\mathrm{n}^{\circ} 713,1993$

[3] Botton S. and al. GPS : localisation et navigation Ouvrage collectif, Hermès, Paris, 1997

[4] Peyret F. Sessyl : the study of positioning systems for road construction robe $12^{\text {th }}$ ISARC, Warsaw, pp. 55-62, June 1995 